\title{
Layered Double Hydroxide
} Nanomaterials Encapsulating Angelica gigas Nakai Extract for Potential Anticancer Nanomedicine

\section{OPEN ACCESS}

Edited by:

Chien-Wen Chang,

National Tsing Hua University, Taiwan

Reviewed by:

Yuet-Kin Leung,

University of Cincinnati, United States

Jui-Yang Lai,

Chang Gung University, Taiwan

Yongsung Hwang,

Soonchunhyang University,

South Korea

*Correspondence:

Tae-il Kim

seal1004@snu.ac.kr

Jae-Min Oh

jaemin.oh@yonsei.ac.kr

tThese authors have contributed equally to this work.

Specialty section:

This article was submitted to Pharmacology of Anti-Cancer Drugs,

a section of the journal

Frontiers in Pharmacology

Received: 12 February 2018

Accepted: 14 June 2018

Published: 09 July 2018

Citation:

Kim H-J, Lee GJ, Choi A-J, Kim T-H, Kim T-i and Oh J-M (2018)

Layered Double Hydroxide

Nanomaterials Encapsulating Angelica gigas Nakai Extract for

Potential Anticancer Nanomedicine.

Front. Pharmacol. 9:723.

doi: 10.3389/fphar.2018.00723

\begin{abstract}
Hyoung-Jun Kim ${ }^{1 \dagger}$, Gyeong Jin Lee ${ }^{2 \dagger}$, Ae-Jin Choi ${ }^{3}$, Tae-Hyun Kim ${ }^{1,4}$, Tae-il Kim ${ }^{2,5 *}$ and
\end{abstract} Jae-Min $\mathrm{Oh}^{1 *}$

${ }^{1}$ Department of Chemistry and Medical Chemistry, College of Science and Technology, Yonsei University, Wonju, South Korea, ${ }^{2}$ Department of Biosystems \& Biomaterials Science and Engineering, College of Agriculture and Life Sciences, Seoul National University, Seoul, South Korea, ${ }^{3}$ Department of Agrofood Resources, National Institute of Agricultural Sciences of RDA, Wanju, South Korea, ${ }^{4}$ Department of Physics, Chemistry and Pharmacy, University of Southern Denmark, Odense, Denmark, ${ }^{5}$ Research Institute of Agriculture and Life Sciences, Seoul National University, Seoul, South Korea

We prepared hybrids consisting of Angelica gigas Nakai (AGN) root or flower extract and layered double hydroxide (LDH) for potential anticancer nanomedicine, as decursin species (DS) in AGN are known to have anticancer activity. Dimethylsulfoxide solvent was determined hybridization reaction media, as it has affinity to both AGN and LDH moiety. In order to develop inter-particle spaces in LDH, a reversible dehydrationrehydration, so-called reconstruction route, was applied in AGN-LDH hybridization. Quantitative analyses on AGN-LDH hybrids indicated that the content of DS was two times more concentrated in the hybrids than in extract itself. Using X-ray diffraction, FT-IR spectroscopy, scanning electron microscopy, and zeta-potential measurement, we found that AGN extract moiety was incorporated into inter-particle spaces of LDH nanoparticles during the reconstruction reaction. Time-dependent DS release from hybrids at $\mathrm{pH} 7.4$ (physiological condition) and pH 4.5 (lysosomal condition) exhibited a $\mathrm{pH}$-dependent release of extract-incorporated LDH hybrids. An anticancer activity test using HeLa, A549, and HEK293T cells showed that the AGN-LDH hybrid, regardless of extract type, showed enhanced anticancer activity compared with extract alone at an equivalent amount of DS, suggesting a nanomedicine effect of AGN-LDH hybrids.

Keywords: layered double hydroxide, Angelica gigas Nakai, anticancer activity, decursin species, nanobiohybrid, phytochemical

\section{INTRODUCTION}

Herbal medicine containing diverse bio-active phytochemicals have long been utilized (Kesarwani and Gupta, 2013; Baumann, 2014). Recently, medicinal researchers have tried to find out therapeutic single component (Lim et al., 2001); however, several studies suggested that whole extract rather than single component is advantageous in therapies as complicated components stimulate multiple targets, induce synergetic effect and act as bio-enhancers (Hu et al., 2009). Therefore, improved drug efficacy could be envisaged with the less-purified extract (Lee et al., 2009; Rasoanaivo et al., 2011).

Angelica gigas Nakai (AGN), one of the herbal crops widely distributed in eastern Asia, has long been utilized as a herbal medicine source. Its root extract in particular has been utilized to 
treat anemia, hypertension, arthritis, menstrual disorders, etc. (Suzuki et al., 2002; Song et al., 2004; Circosta et al., 2006; Guo et al., 2007; Kil et al., 2008). According to recent researches, roots, stems, and leaves of AGN contain bio-active substances such as pyranocoumarins (decursin, decursinol angelate, prantschimgin, angelinol, agasyllin, etc.), flavonoids, polysaccharides, phenolics, and volatile aromatics (Zhang et al., 2012). We verified in our previous study that the flowers of AGN, which have not been utilized previously as a herbal medicine resource, are also rich in antioxidants, such as phenolic acids and flavonoids (Kim et al., 2015). Among those phytochemicals, the decursin species (DS: decursin and decursinol angelate) have been shown to have excellent anticancer activity in prostate cancer (Jiang et al., 2006; Guo et al., 2007). Similar to other herbal medicine-based studies, DS administered with other extract components showed higher anticancer efficacy on lung and prostate cancers compared with DS only (Lee et al., 2009).

In this sense, a natural extract containing bio-active substances and bio-enhancers is expected to show improved drug efficacy over purified single molecules. However, there are still problems with herbal medicine in that the content of active substances responsible for the major functions is very low. According to our previous study, a methanol extract of AGN root contains only $8.5 \mathrm{wt} / \mathrm{wt} \%$ of antioxidant (phenolic acid and flavonone) and 0.2 $w t / w t \%$ of anticancer DS species (Kim et al., 2016). Thus, a high dose of AGN extract would be required for practical application.

In order to overcome these drawbacks, strategies of nanomedicine could be taken. Nanomaterials such as liposomes, polymeric particles, dendrimers, and inorganic materials have been utilized as nanomedicine vehicles. They can encapsulate drugs using their appropriate internal spaces and adsorb drugs on large surface to protect vulnerable molecules from external harsh condition (Oh et al., 2006b). They can rearrange drug molecules in their interior to modify solubility (Mohammed et al., 2004), to improve bioavailability and to release drug in controlled manner (Maincent et al., 1986; Kang et al., 2015). Especially, $\mathrm{pH}$ responsive drug release is possible in several vehicles utilizing nanomaterials' pH-dependent properties (Kim et al., 2007). Several nanomaterials were developed to deliver drug molecules to target organs using surface modified nanomaterials as targeting ligands (Oh et al., 2009). In this way, nanomedicine can reduce the administration dose of drug molecules. Among various nanomaterials, layered double hydroxides (LDHs), which can characteristically encapsulate bio-active molecules in their 2-dimensional interlayer space, are potential drug delivery carriers. LDHs have a layer-by-layer stacked structure consisting of a positive layer $\left(\mathrm{M}^{2+}{ }_{1-\mathrm{x}} \mathrm{M}^{3+}{ }_{\mathrm{x}}(\mathrm{OH})_{2}{ }^{\mathrm{x}+}: \sim 0.5 \mathrm{~nm}\right.$ thickness, hundreds of nanometers in diameter) and exchangeable anions $\left(\mathrm{A}^{\mathrm{n}-\mathrm{x} / \mathrm{n}}\right)$ (Rives, 2001). Interestingly, the interlayer space of LDHs can be as small as $0.37 \mathrm{~nm}$ with an interlayer of $\mathrm{NO}_{3}{ }^{-}$ and be freely expanded to a few nanometers with bulky organic species (Cavani et al., 1991; Rives, 2001). Thus, LDHs have been reported to stabilize fragile biomolecules (Oh et al., 2006b), to solubilize poorly water-soluble drugs (Berber et al., 2008), to release incorporated drug moieties in a controlled manner (Kang et al., 2015), and to transport massive amounts of drug molecules into intracellular systems (Oh et al., 2006c). LDHs have several more characteristic advantages as delivery carriers. LDHs are mechanically more stable than organic-based carriers like liposomes. They are less cytotoxic and more biocompatible than the other nanoparticles such as iron oxides and carbon nanotubes (Choi et al., 2009). They can be dissolved into simple ions at weakly acidic condition (possibly lysosomal condition), while organic carriers produce various sized residues upon hydrolysis. In terms of natural extract incorporation, LDHs can be effective in that (i) their interlayer nanospace and large inter-particle space provide appropriate spaces for variously sized natural extract molecules (Rives, 2001; Kang et al., 2015), and (ii) positive layer charges have a high affinity for phytochemicals, which usually have a negative charge or $\delta^{-}$sites (Rives, 2001; Kim et al., 2016).

In this study, we encapsulated whole natural extract from the root or flower of AGN into $\mathrm{LDH}$ nanomaterials. We tried to incorporate most of the phytochemical substances of the natural extracts into $\mathrm{LDH}$ nanomaterials, considering the bio-enhancer function. In our previous study, we showed the possibility to load extract moiety into LDH hybrids (Kim et al., 2016). However, that hybrid contained quite low amount of active components (DS), and thus it is strongly required to enhance DS content for practical applications as anticancer nanomedicine. In this regard, we designed encapsulation conditions to optimally concentrate DS in LDH nanomedicine. Solvent conditions were carefully selected in order to solubilize both hydrophilic and hydrophobic natural extract molecules and to facilitate the incorporation of the organic moieties such as carbohydrates, lipids, flavonoids, and DS in AGN extract into LDH nanomaterials. The encapsulation method was strategically chosen to maximize incorporation efficiency and to enhance the affinity for cellular membranes. LDHs can undergo a reversible dehydration-rehydration process, called reconstruction (Hibino and Tsunashima, 1998). Thermal treatment of $\mathrm{LDH}$ nanomaterials at $\sim 400^{\circ} \mathrm{C}$ produces a mixed metal oxide that can recover the original $\mathrm{LDH}$ structure in the presence of water and anionic species to be encapsulated (Nyambo et al., 2009). This encapsulation strategy forms particle nanostructures that are less crystalline and highly interconnected, resulting in inter-particle space, which can accommodate various organic substances (Kang et al., 2015), while the external surface of an organic-LDH hybrid possesses a positive charge for effective interactions with negative cellular membrane (Oh et al., 2006a). The goal of this study is to investigate the structure of the obtained hybrid nanomedicine and to evaluate their potential for anticancer treatment. In this regard, DS concentration effect and controlled release by LDH nanomaterials were investigated and the anticancer properties of the hybrid nanomedicines were evaluated in several cancer cells.

\section{MATERIALS AND METHODS}

\section{Materials}

Root and flower extracts of Angelica gigas Nakai (AGN) were provided by the National Institute of Horticultural and Herbal Science in Korea. Magnesium nitrate hexahydrate $\left[\mathrm{Mg}\left(\mathrm{NO}_{3}\right)_{2}\right.$. $\left.6 \mathrm{H}_{2} \mathrm{O}\right]$, aluminum nitrate nonahydrate $\left[\mathrm{Al}\left(\mathrm{NO}_{3}\right)_{3} \cdot 9 \mathrm{H}_{2} \mathrm{O}\right]$, 
sodium bicarbonate $\left(\mathrm{NaHCO}_{3}\right)$, and dimethylsulfoxide (DMSO) were purchased from Sigma-Aldrich Co. LLC. (St. Louis, MO, United States). A standard decursin sample for chromatography was purchased from ChromaDex Inc. (Irvine, CA, United States). Sodium hydroxide $(\mathrm{NaOH})$, n-hexane, and chloroform were acquired from DAEJUNG CHEMICALS \& METALS CO. LTD. (Siheung, South Korea). Methanol (Burdick \& Jackson ${ }^{\circledR}$, Morristown, NJ, United States) and acetone (SAMCHUN CHEMICAL CO., Yeosu, South Korea) were used to solubilize AGN extract solid. Hanks' balanced salt solution (HBSS) was obtained from WELGENE Inc. (Taipei, Taiwan). Phosphate buffered saline (PBS) was acquired from Lonza (Walkersville, $\mathrm{MD}$, United States). All reagents were used without further purification.

\section{Solubility of DS in Various Solvents}

Dried AGN extract (200 mg), which was previously extracted in methanol at $25^{\circ} \mathrm{C}$ and dried by vacuum evaporation at $60^{\circ} \mathrm{C}$, was dissolved in six different solvents $(100 \mathrm{~mL})$ including deionized water (DW), methanol, DMSO, acetone, n-hexane, and chloroform. The DS content in each solution was quantified by high performance liquid chromatography (HPLC, Yonglin YL9100 Series, Anyang, South Korea). The mobile phase was an acetonitrile:DW (6:4) mixture with a flow rate of $0.8 \mathrm{~mL} / \mathrm{min}$, and a UV-Vis detector with a wavelength of $350 \mathrm{~nm}$ was used.

\section{Preparation of Pristine LDH (MgAl-LDH) and Its Calcined Form}

The pristine MgAl-LDH was prepared by a conventional coprecipitation method followed by hydrothermal treatment, as reported elsewhere (Kim et al., 2011). $\mathrm{Mg}\left(\mathrm{NO}_{3}\right)_{2} \cdot 6 \mathrm{H}_{2} \mathrm{O}$ $(0.06 \mathrm{~mol})$ and $\mathrm{Al}\left(\mathrm{NO}_{3}\right)_{3} \cdot 9 \mathrm{H}_{2} \mathrm{O}(0.03 \mathrm{~mol})$ were dissolved in $200 \mathrm{~mL}$ of $\mathrm{DW}$, and the solution was titrated to $\mathrm{pH} \sim 9.5$ with $\mathrm{NaOH} / \mathrm{NaHCO}_{3}(0.23 \mathrm{~mol} / 0.17 \mathrm{~mol}$ in $250 \mathrm{~mL} \mathrm{DW})$. Then, the white suspension was transferred to a Teflon-lined autoclave and put in an oven at $150^{\circ} \mathrm{C}$ for $24 \mathrm{~h}$. Finally, a white precipitate (MgAl-LDH) was collected by centrifugation, thoroughly washed with DW, and then lyophilized. In order to prepare the calcined $\mathrm{LDH}$, the MgAl-LDH powder was put in an alumina boat and thermally treated at $400^{\circ} \mathrm{C}$ in an electric muffle furnace for $9 \mathrm{~h}$.

\section{Preparation of AGN-LDH Hybrid by Reconstruction}

AGN extracts, both from root (RT) and flower (FL) (27.1 $\mathrm{mg}$ and $36.6 \mathrm{mg}$, respectively), were dissolved in $1 \mathrm{~mL}$ of DMSO, and then $30 \mathrm{~mL}$ of calcined $\mathrm{LDH}$ in water suspension $(23 \mathrm{mg} / \mathrm{mL})$ was added to the AGN/DMSO solutions. After thorough mixing, 70 and $44 \mathrm{~mL}$ of $\mathrm{DW}$ was added to the mixtures for $\mathrm{RT}$ and FL, respectively, to reconstruct the $\mathrm{LDH}$ structure. The reactants were stirred for $12 \mathrm{~h}$ in darkness. Then, the suspension was centrifuged, washed with DW, and lyophilized. The obtained hybrids were designated as RT-LDH and FL-LDH for AGN root extract-incorporated hybrid and flower extract-incorporated hybrid, respectively.

\section{Characterization}

The crystal structures of MgAl-LDH, its calcined form, and the hybrids were investigated by powder X-ray diffraction (PXRD, D2 Phaser with LYNXEYE ${ }^{\mathrm{TM}}$ detector, Bruker AXS GmbH, Kalsruhe, Germany) with $\mathrm{Cu} \mathrm{K}_{\alpha}$ radiation $(\lambda=1.5406 \AA$ ). The ground powder samples were mounted on a poly(methyl methacrylate) holder, and a diffraction pattern was obtained in the $2 \theta$ range of $3-80^{\circ}$ with scanning steps of $0.02^{\circ}$. The chemical property of $\mathrm{MgAl}-\mathrm{LDH}, \mathrm{AGN}$ extract and the hybrids was confirmed by Fourier transform-infrared spectroscopy (FT-IR, spectrum one B.v5.0 spectrometer, Perkin Elmer, Waltham, MA, United States) utilizing conventional $\mathrm{KBr}$ method. The morphology and particle size of MgAl-LDH, its calcined form, and the hybrids were observed with field emission-scanning electron microscopy (FE-SEM, Quanta 250 FEG, FEI, Hillsboro, OR, United States). For the SEM measurement, the powder samples were immobilized on carbon tapes and sputtered with $\mathrm{Pt} / \mathrm{Pd}$ for $30 \mathrm{~s}$. For the zeta-potential measurement, $1 \mathrm{mg}$ of MgAl-LDH and each hybrid powder were dispersed in $1 \mathrm{~mL} \mathrm{DW}$, and then the zeta-potential was measured by ELSZ-1000 (Otsuka, Kyoto, Japan). The contents of AGN (RT or FL) in corresponding hybrids were evaluated by measuring the weight difference of the organic moiety before and after the hybridization reaction. DS was quantified with HPLC as described above.

\section{DS Release Study}

In order to evaluate the amount of DS released from the hybrids, $50 \mathrm{mg}$ of hybrid powder was dispersed into $100 \mathrm{~mL}$ of media such as $\mathrm{pH} 7.4 \mathrm{PBS}$ or $\mathrm{pH} 4.5 \mathrm{HBSS}$. At the determined time point $(5,10,15,20,25,30,60,90$, or $120 \mathrm{~min}), 1 \mathrm{~mL}$ of suspension was collected, and the solid part was removed by centrifugation at $8000 \mathrm{rpm}$ and filtration with a $0.45-\mu \mathrm{m}$ pore syringe filter (Advantec Toyo Roshi Kaisha Ltd., Tokyo, Japan). The fractional release of DS was quantified with an HPLC, as described above. All experiments were performed in triplicate. The observed time-dependent release was fitted with four kinds of kinetic models including the first-order kinetic (Eq. 1), Elovich model (Eq. 2), parabolic diffusion (Eq. 3), and power function (Eq. 4), as shown below (Jalali, 2006; Shariatmadari et al., 2006).

$$
\text { First order } \ln Q_{\mathrm{t}}=\ln Q_{\mathrm{e}}-\mathrm{k}_{1} t
$$

$\mathrm{k}_{1}$ : first-order rate constant $\left(\mathrm{h}^{-1}\right)$

$$
\text { Elovich equation } Q_{\mathrm{t}}=a+b \ln t
$$

$a$ : released quantity in the initial phase, $b$ : release rate

$$
\text { Parabolic diffusion } Q_{\mathrm{t}}=Q_{\mathrm{e}}+R \mathrm{t}^{1 / 2}
$$

$R$ : diffusion rate constant

$$
\text { Power function } Q_{t}=\alpha t^{\beta}
$$

$\alpha$ : initial desorption rate constant, $\beta$ : desorption rate coefficient

$Q_{\mathrm{t}}$ is the released amount after $t \mathrm{~min}$, and $Q_{\mathrm{e}}$ is the released amount at equilibrium for all models. 


\section{Cell Culture}

Human cervical adenocarcinoma cells (HeLa), human lung adenocarcinoma epithelial cells (A549), human embryonic kidney cells containing SV40 Large T-antigen (HEK293T), and mouse myoblast cells (C2C12) were grown in DMEM supplemented with $10 \%$ FBS and $1 \%$ penicillin/streptomycin in a humidified atmosphere containing $5 \% \mathrm{CO}_{2}$ at $37^{\circ} \mathrm{C}$.

\section{Trypan Blue Assay of AGN Extracts and AGN-LDH Hybrids}

Anticancer activity of AGN extract and AGN-LDH hybrids was evaluated by trypan blue assay. Conventional MTT assay was not used because DS can disturb the absorbance of formazan in MTT assay. Cells were seeded at a density of $2.0 \times 10^{5}$ cells per well on 6-well culture plates. After $24 \mathrm{~h}$ of incubation, sample solutions or suspensions (0.1\% DMSO/serum-containing DMEM) were added to the cells at pre-determined DS concentrations for $24 \mathrm{~h}$. Then, cells were trypsinized and collected in PBS. After centrifugation (100 g, $5 \mathrm{~min}$ ), cell pellets were resuspended in $1 \mathrm{~mL} \mathrm{PBS}$ and stained with $0.4 \%$ trypan blue solution for $2 \mathrm{~min}$ at room temperature (Strober, 2001). The numbers of stained dead cells and unstained viable cells among 200-300 cells were counted with a hemacytometer each time. All experiments were performed in triplicate.

\section{RESULTS}

\section{Solubility of DS in Various Solvents}

Table 1 displays the solubility of extracts and DS (Figure 1) in various solvents. Dried AGN methanol extract was dissolved in several solvents: polar protic solvents (water, methanol), polar aprotic solvents (DMSO, acetone), and non-polar solvents (n-hexane, chloroform) (Figure 1). In terms of dissolution efficacy for total extract components, methanol, DMSO, and deionized water showed excellent capacities. However, water did not effectively solubilize DS. Considering both total extract and DS dissolution, DMSO showed the best ability and thus was selected as the hybridization reaction media.

\section{Characterization of AGN-LDH Hybrids}

The crystal structures of the two AGN-LDH hybrids, RT-LDH and FL-LDH, were analyzed and compared with those of pristine

TABLE 1 | Dissolved extract and DS from 200 mg of Angelica gigas Nakai root methanol extract.

\begin{tabular}{lccr}
\hline Solvent type & $\begin{array}{c}\text { Extraction } \\
\text { solvent }\end{array}$ & $\begin{array}{c}\text { Dissolved } \\
\text { extract }(\mathbf{m g})\end{array}$ & $\begin{array}{c}\text { Dissolved } \\
\text { DS (mg) }\end{array}$ \\
\hline Polar protic & Deionized water & $137 \pm 39$ & $2.5 \pm 0.01$ \\
& Methanol & $202 \pm 24$ & $40.3 \pm 0.54$ \\
Polar aprotic & Acetone & $76 \pm 8.9$ & $34.1 \pm 0.59$ \\
& Dimethylsulfoxide & $192 \pm 25$ & $58.4 \pm 7.48$ \\
Non-polar & n-Hexane & $57 \pm 11$ & $31.5 \pm 0.62$ \\
& Chloroform & $61 \pm 17$ & $27.2 \pm 0.68$ \\
\hline
\end{tabular}

DS, decursin species.
$\mathrm{LDH}$ and the extract itself by measuring powder X-ray diffraction patterns (Figure 2). Pristine LDH showed sharp diffraction peaks at $2 \theta$ values of $11.7,23.5,34.6,38.5,45.5,60.8$, and $62.2^{\circ}$, which could be assigned to (hkl) indexes of (003), (006), (012), (015), (018), (110), and (113), respectively, showing the intended hydrotalcite (JCPDS (Joint Committee on Powder Diffraction Standards) card No. 14-0191) phase. Calcined LDH exhibited (200) and (220) peaks at $2 \theta 43.3$ and $62.5^{\circ}$, suggesting the evolution of $\mathrm{MgO}$ (periclase, JCPDS card No. 45-0946) upon thermal treatment. In contrast, two kinds of extracts from RT and FL showed an amorphous phase with some salt-like peaks in XRD patterns. After hybridization, the crystal structure of LDH was observed to be recovered, showing the (003), (006), (012), (018), and (110) peaks of hydrotalcite. The decreased crystallinity was attributed to random stacking of $\mathrm{LDH}$ nanolayers and large organic moieties, implying the incorporation of extract inside the LDH nanostructures (Kim et al., 2016).

The FT-IR spectrum of RT extract showed the characteristic bands (dashed arrows) of polysaccharide and DS such as $\alpha$-pyrone ring $\left(1717 \mathrm{~cm}^{-1}\right)$, aromatic $\mathrm{C}=\mathrm{C} \quad(1632$ and $\left.1566 \mathrm{~cm}^{-1}\right)$, C-O stretching $\left(1226 \mathrm{~cm}^{-1}\right)$ and aromatic C-O (1136 and $1028 \mathrm{~cm}^{-1}$ ) (Figure 3A) (Li et al., 2014; Kim et al., 2016). The characteristic peak of MgAl-LDH (solid arrow) was exhibited at $1365 \mathrm{~cm}^{-1}$ (stretching vibration of interlayer $\mathrm{CO}_{3}{ }^{2-}$ ) and $790,674 \mathrm{~cm}^{-1}$ (both for $\mathrm{M}-\mathrm{OH}$ stretching of $\mathrm{LDH}$ ). After hybridization, all characteristic bands of RT extract and MgAl-LDH were observed at similar peak position with RT and $\mathrm{MgAl}-\mathrm{LDH}$. In case of FL extract, - $\mathrm{OH}$ bending of polysaccharide and aromatic C-O stretching of DS were exhibited at $1664 \mathrm{~cm}^{-1}$ and $1117,1029 \mathrm{~cm}^{-1}$, respectively (Figure 3B). IR spectrum of FL-LDH hybrid indicated that the characteristic peak of FL and $\mathrm{MgAl}-\mathrm{LDH}$ were also preserved after hybridization.

Scanning electron microscopic (SEM) images showed step-by-step morphological changes of $\mathrm{LDH}$ from pristine to AGN-LDH hybrids. Pristine LDH (Figure 4a) exhibited particles having a thick coin-like shape with thickness of $\sim 90 \mathrm{~nm}$ and a diameter of $\sim 244 \mathrm{~nm}$, which corresponded to the hydrodynamic radius, $255 \mathrm{~nm}$, of MgAl-LDH (Supplementary Figure S1A). Upon calcination, the thickness of the particle was reduced to $\sim 50 \mathrm{~nm}$, while the diameter and shape were mostly maintained (Figure 4b). After hybridization, the overall morphology of particles changed significantly. Both RT-LDH and FL-LDH hybrids demonstrated sand-rose shapes, in which very thin layers were partially bent and agglomerated (Figures $\mathbf{4 c , d}$ ). Although the images for both hybrids seemed to have assembly of particles, close observation exhibited that the primary particle size of pristine LDH, RT-LDH, and FL-LDH hybrid were comparable to each other showing 244, 241, and $239 \mathrm{~nm}$ (dashed circles in Figure 4), respectively. The hydrodynamic radii of both hybrids in aqueous suspension were 595 and $826 \mathrm{~nm}$ for RT-LDH and FL-LDH (Supplementary Figures S1B,C), respectively, suggesting the possibility of agglomerate with two or three particles in hybrids.

Surface charges of pristine LDH, AGN extract, and hybrids were investigated by measuring their zeta potentials in an aqueous media of $\mathrm{pH} \sim 7.0$. As shown in Figure 5, pristine $\mathrm{LDH}$ was determined to have a high positive surface charge of 


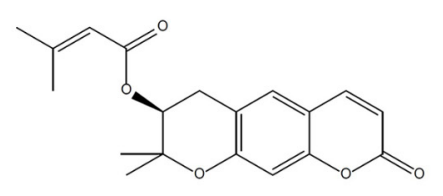

Decursin

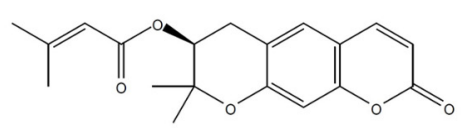

Decursinol angelate

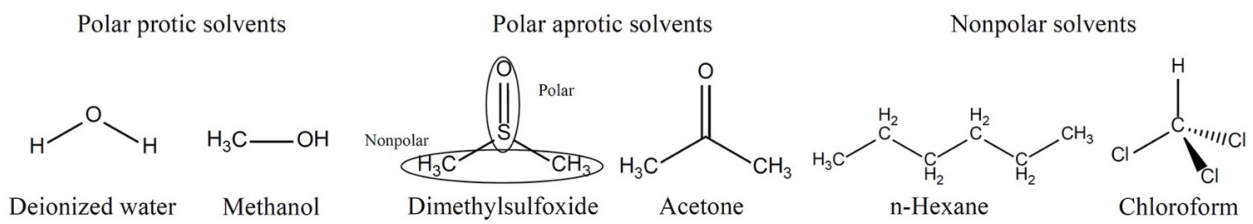

FIGURE 1 | Molecular structures of DS and secondary extraction solvents. DS, decursin species.

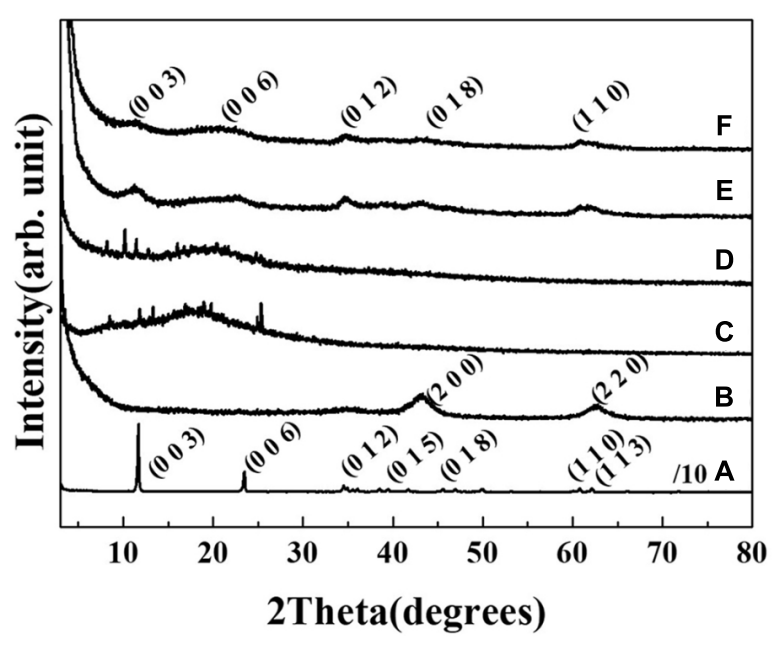

FIGURE 2 | Powder X-ray diffraction patterns of (A) MgAl-LDH, (B) calcined $\mathrm{LDH}, \mathbf{( C )}$ root extract powder, (D) flower extract powder, (E) RT-LDH hybrid, and (F) FL-LDH hybrid. LDH, layered double hydroxide; RT, root; FL, flower.

$\sim+38.5 \mathrm{mV}$, and the distribution of zeta potential lay in the range of $+10 \sim+75 \mathrm{mV}$ (solid line). Different from pristine LDH, zeta potential values of both AGN extracts (RT and FL) showed strong negative charges centered at $\sim-75.6 \mathrm{mV}$, and the distribution was from -50 to $-130 \mathrm{mV}$. After hybridization, zeta potential value again shifted to the positive region. For both hybrids, the distribution lay between 0 and $+50 \mathrm{mV}$ with an average value of $+26.7 \mathrm{mV}$.

Table 2 displays the DS quantification results of the hybrids. First, the extract content was 18.4 and $16.1 \mathrm{wt} / \mathrm{wt} \%$ for RT-LDH and FL-LDH hybrids, respectively. The content of DS was 11.5 and $2.23 \mathrm{wt} / \mathrm{wt} \%$ for RT-LDH and FL-LDH hybrids, respectively. DS content in the original extracts was 30.8 and $6.27 \mathrm{wt} / \mathrm{wt} \%$ in AGN root and flower, respectively. When we estimated DS content in total extract inside hybrids, they were much larger than those in the original extract, $62.8 \mathrm{wt} / \mathrm{wt} \%$ and $13.8 \mathrm{wt} / \mathrm{wt} \%$ for RT-LDH and FL-LDH, respectively.

\section{Evaluation on DS Release From Hybrids}

Time-dependent release profiles of DS from hybrids in either neutral physiological condition ( $\mathrm{pH} 7.4$ PBS) or lysosomal condition ( $\mathrm{pH} 4.5$ HBSS) are displayed in Figure 6. RT-LDH slowly released the DS in pH 7.4 PBS, and the cumulative release reached $\sim 6.1 \%$ after $120 \mathrm{~min}$ (Figure 6A). In contrast to the release profile in $\mathrm{pH} 7.4 \mathrm{PBS}$, RT-LDH in $\mathrm{pH}$ 4.5 HBSS showed fast release at the early stage (0-25 $\mathrm{min})$, followed by slow and sustained release. The cumulative DS release was almost 35\% at $120 \mathrm{~min}$, and the initial burst of DS before $25 \mathrm{~min}$ was responsible for $\sim 30 \%$ of the total release (Figure 6A). The FL-LDH hybrid showed similar patterns with RT-LDH both in $\mathrm{pH}$ 7.4 PBS and pH 4.5 HBSS with more suppressed DS release (Figure 6B). The cumulative DS release in the early stage before 25 min was $\sim 1.0$ and $11 \%$ in $\mathrm{pH} 7.4 \mathrm{PBS}$ and $\mathrm{pH} 4.5 \mathrm{HBSS}$, and the cumulative release at 120 min was $\sim 2.1$ and $18 \%$, respectively.

The release profiles were fitted to several well-established kinetic models, such as first order, Elovich, parabolic, and power function equations, and their fitting results are summarized in Table 3. DS release patterns of RT-LDH and FL-LDH hybrids in $\mathrm{pH} 7.4 \mathrm{PBS}$ and $\mathrm{pH}$ 4.5 HBSS were well-fitted to the Elovich model, showing coefficient of determination $R^{2}$ values larger than 0.98 except pH RT-LDH in pH 4.5 HBSS. Although RT-LDH in pH 4.5 HBSS media showed a relatively lower $\mathrm{R}^{2}$ value of 0.8451 , the Elovich model was determined to be the most reasonable kinetic model among the fitted models in terms of $R^{2}$ value.

The Elovich parameters, $a$ and $b$, for two AGN-LDH hybrids in PBS and HBSS conditions are summarized in Table 4. Parameter $a$ is related to the release in the initial phase; a higher $a$ value is related to a larger amount of initial release (Mengel, 2000). Parameter $b$ stands for the release rate of a loaded moiety (Jalali, 2006). RT-LDH showed an initial burst effect in $\mathrm{pH} 4.5$ HBSS $(a=6.21)$, while its release in the early stage was suppressed in $\mathrm{pH} 7.4$ PBS $(a=-0.0539)$. The release rate of RT-LDH was higher in HBSS condition $(b=3.26)$ than in the PBS $(b=0.738)$. For FL-LDH, the parameter $a$ was similar to each other in both types of release media, while the release rate of FL-LDH was higher in $\mathrm{pH} 4.5 \mathrm{HBSS}(b=0.509)$ than in the $\mathrm{pH} 7.4$ PBS $(b=0.00784)$. Compared with RT-LDH, the initial DS release 

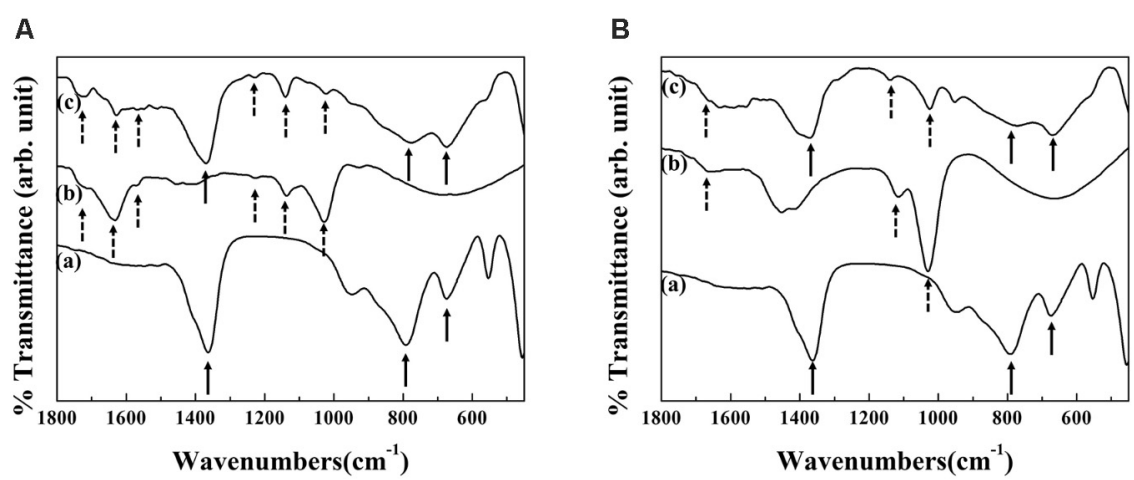

FIGURE 3 | Fourier transform-infrared spectra of (a) MgAl-LDH, (b) extract and (c) hybrid for (A) RT-LDH and (B) FL-LDH hybrid. LDH, Layered double hydroxide; DS, Decursin species; RT, Root; FL, Flower.
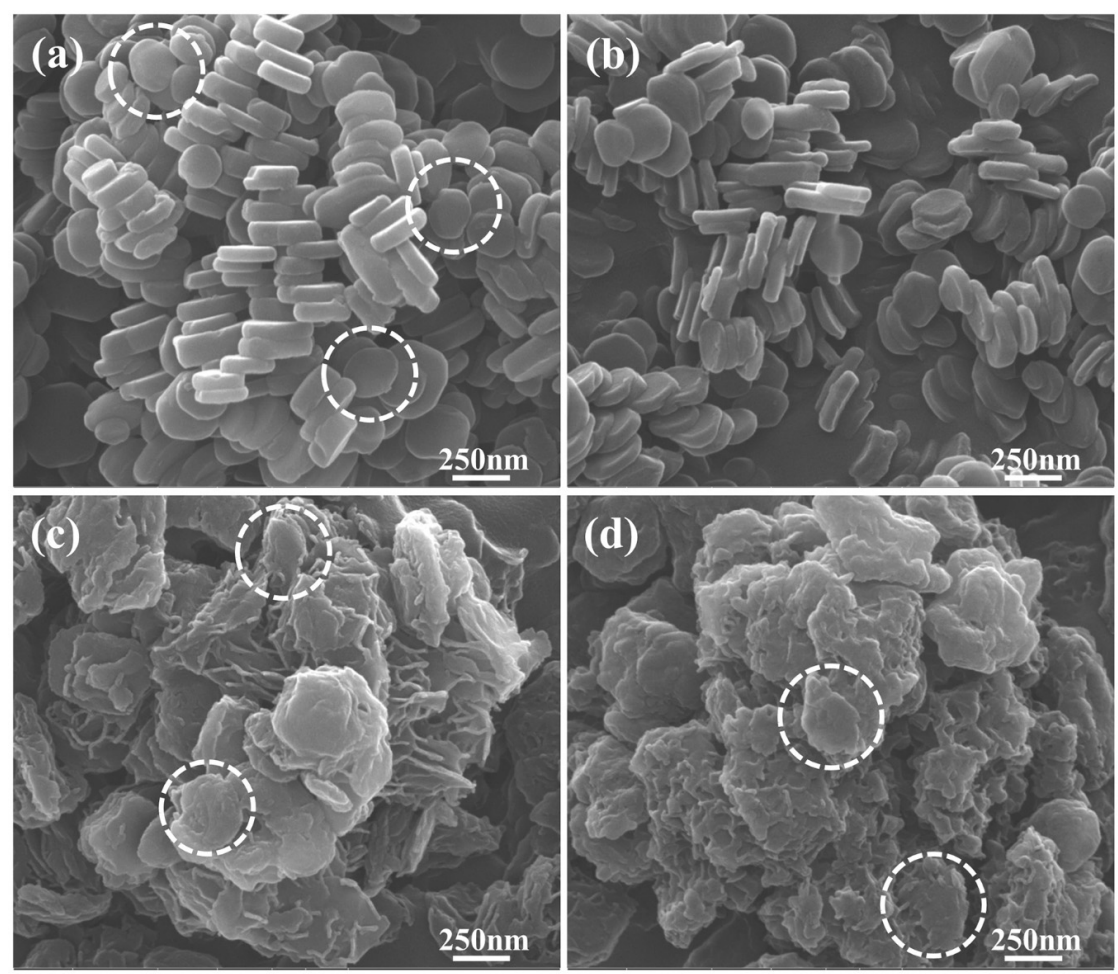

FIGURE 4 | Scanning electron microscopic images of (a) MgAl-LDH, (b) calcined LDH, (c) RT-LDH hybrid, and (d) FL-LDH hybrid. Dotted circles stand for primary particles. LDH, layered double hydroxide; RT, root; FL, flower.

from FL-LDH was fairly suppressed $(a=6.21$ and -0.407 for RT-LDH and FL-LDH in HBSS media, respectively), and the release rate was very slow $(b=0.00784$ and 0.509 for PBS and HBSS).

\section{Anticancer Efficacy of AGN-LDH Hybrids}

Anticancer efficacy of AGN-LDH hybrids was examined in A549, HeLa, and HEK293T cells by trypan blue assay. C2C12 myoblast cells were used as a control. Hybrid sample suspensions were treated based on DS concentration, which was calculated according to the pre-quantified DS content in hybrids. According to the preliminary test, $0.1 \%$ DMSO/DMEM solution was found to be non-cytotoxic. As shown in Figure 7, AGN extracts from both FL and RT displayed low cancer cell-killing activities. Less than $10 \%$ of A549 cells and somewhat more HeLa and HEK293T cells (about 10-20\%) were found to be dead at a high DS concentration of $50 \mu \mathrm{M}$. However, DS-containing hybrids (FL-LDH and RT-LDH) exhibited much higher cancer cellkilling activities in all cell types than did the AGN extract. Above $20 \mu \mathrm{M}$, more cancer cells were found to be dead in the hybrid-treated condition than in the AGN extract only treated condition. Furthermore, RT-LDH showed a higher anticancer 

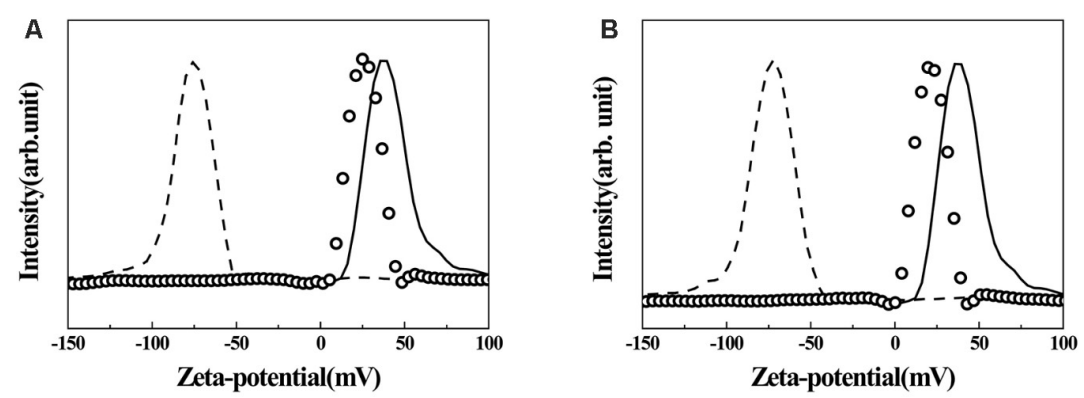

FIGURE 5 | Zeta-potential graphs of AGN-LDH hybrids. (A) RT-LDH hybrid and (B) FL-LDH hybrid. Solid line: MgAl-LDH, dashed line: AGN extract, open circles: AGN-LDH hybrids. AGN, Angelica gigas Nakai; LDH, layered double hydroxide; RT, root; FL, flower.

TABLE 2 | Loading capacity and DS content for AGN extract and DS in AGN-LDH hybrids.

\begin{tabular}{lccc}
\hline & $\begin{array}{c}\text { Extract loading } \\
\text { capacity in hybrid } \\
\text { [extract/hybrid } \\
\text { (wt/wt\%)] }\end{array}$ & $\begin{array}{c}\text { DS loading } \\
\text { capacity in hybrid } \\
\text { [DS/hybrid } \\
\text { (wt/wt\%) }\end{array}$ & $\begin{array}{c}\text { DS content in } \\
\text { total extract } \\
\text { [DS/extract } \\
\text { (wt/wt\%)] }\end{array}$ \\
\hline Root extract & - & - & $30.8 \pm 0.12$ \\
Flower extract & - & - & $6.27 \pm 0.13$ \\
RT-LDH & $18.4 \pm 1.28$ & $11.5 \pm 0.05$ & $62.8 \pm 4.28$ \\
FL-LDH & $16.1 \pm 0.08$ & $2.23 \pm 0.004$ & $13.8 \pm 4.73$
\end{tabular}

AGN, Angelica gigas Nakai; DS, decursin species; LDH, layered double hydroxide; $R T$, root; $F L$, flower.

activity than FL-LDH in all cell types, with about $40-50 \%$ of cancer cells killed at $50 \mu \mathrm{M}$ in the RT-LDH-treated condition. Interestingly, RT-LDH, and FL-LDH showed low dead cells population $(<20 \%$, even at high concentration of $50 \mathrm{mM} \mathrm{DS})$ in C2C12 cells, similarly with root and flower extract (Figure 7D).

\section{DISCUSSION}

According to the solubility test (Table 1), the organic moieties in the AGN extract including DS can be effectively dissolved utilizing polar aprotic DMSO (Figure 1). Amphiphilic DMSO, which has both hydrophobic (two methyl groups) and hydrophilic (sulfoxide group) groups, was expected to effectively dissolve both hydrophilic and hydrophobic chemical species in the extracts. Furthermore, DMSO can facilitate effective reactions between hydrophobic DS and hydrophilic LDH nanomaterials. Thus, DMSO was selected as the hybridization media. The extract/DMSO solutions were determined to have 30.8 and 6.27 $\mathrm{wt} / \mathrm{wt} \%$ of DS for AGN root and flower, respectively, providing contents that were sufficiently high to utilize DS as active components in the anticancer study after hybridizing with LDHs.

Physicochemical analyses such as XRD, FT-IR, SEM, and zeta potential measurement showed that the hybrids possessed AGN extract inside the nanostructures formed by LDH nanoparticles. According to XRD patterns (Figure 2), FT-IR spectra (Figure 3), and SEM images (Figure 4), we conclude that the organic moieties in the AGN extract were incorporated into the LDH nanostructure during the reconstruction process. Crystallinity reduction and peak shift to a lower angle in the hybrid compared with in the pristine LDH indicated that the organic moieties were incorporated. Generally, LDHs undergo partial lattice distortion and re-organization during a reconstruction process (PérezRamírez et al., 2007; Martínez-Ortiz Mde et al., 2008), and this distortion and re-organization could be more significant when large organic molecules, such as those from natural extract, were incorporated (Kim et al., 2016). Diffraction patterns of AGN, both amorphous patterns in the $2 \theta$ range $5-30^{\circ}$ and several small and sharp peaks, disappeared after hybridization, confirming that
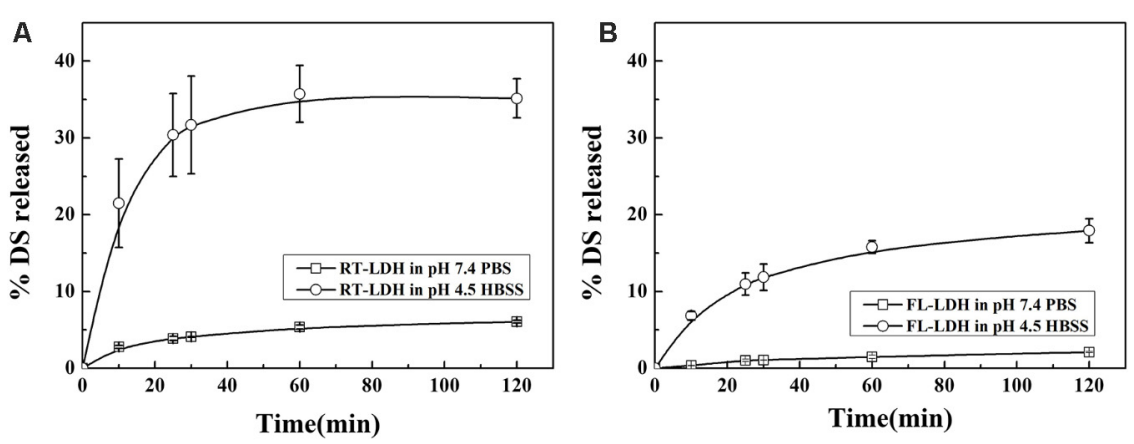

FIGURE 6 | Release patterns of DS from (A) RT-LDH hybrid and (B) FL-LDH hybrid in pH 7.4 PBS and pH 4.5 HBSS. DS, Decursin species; RT, Root; FL, Flower; LDH, Layered double hydroxide; PBS ( $\square$ ), Phosphate buffered saline; HBSS $(\bigcirc)$, Hanks' balanced salt solution. 
TABLE 3 | Linear regression results of time-dependent DS release pattern from RT-LDH and FL-LDH nanohybrids for kinetic model fitting.

\begin{tabular}{|c|c|c|c|}
\hline & Kinetic model & Regression equation & $R^{2}$ \\
\hline \multirow[t]{4}{*}{ RT-LDH/pH 7.4 PBS } & First order & $\operatorname{lnQt}=-1.834 \times 10^{-4} t-0.008964$ & 0.6988 \\
\hline & Elovich equation & $\mathrm{Qt}=0.7376 \ln \mathrm{t}-0.05392$ & 0.9950 \\
\hline & Parabolic diffusion & $\mathrm{Qt}=5.525 \times 10^{-3} t^{1 / 2}+0.007202$ & 0.9338 \\
\hline & Power function & $\operatorname{lnQt}=0.3219 \ln t-0.2413$ & 0.9837 \\
\hline \multirow[t]{4}{*}{ FL-LDH/pH 7.4 PBS } & First order & $\operatorname{lnQt}=-7.173 \times 10^{-5} t-0.001484$ & 0.8832 \\
\hline & Elovich equation & $\mathrm{Qt}=7.842 \times 10^{-2} \operatorname{Int}-0.1416$ & 0.9897 \\
\hline & Parabolic diffusion & $\mathrm{Qt}=2.136 \times 10^{-3} t^{1 / 2}+0.001714$ & 0.9678 \\
\hline & Power function & $\operatorname{lnQt}=0.6956 \operatorname{lnt}-4.614$ & 0.9193 \\
\hline \multirow[t]{4}{*}{ RT-LDH/pH4.5 HBSS } & First order & InQt $=-9.354 \times 10^{-3} t-0.5852$ & 0.5831 \\
\hline & Elovich equation & $\mathrm{Qt}=3.256 \operatorname{lnt}+6.210$ & 0.8451 \\
\hline & Parabolic diffusion & Qt $=4.314 \times 10^{-2} t^{1 / 2}+0.5687$ & 0.6766 \\
\hline & Power function & $\operatorname{lnQt}=0.1992 \ln t+2.154$ & 0.8132 \\
\hline \multirow[t]{4}{*}{ FL-LDH/pH4.5 HBSS } & First order & InQt $=-2.105 \times 10^{-3} t-0.1221$ & 0.8898 \\
\hline & Elovich equation & Qt $=0.5092 \mathrm{lnt}-0.4070$ & 0.9920 \\
\hline & Parabolic diffusion & $\mathrm{Qt}=4.330 \times 10^{-2} t^{1 / 2}+0.1127$ & 0.9368 \\
\hline & Power function & $\operatorname{lnQt}=0.3917 \operatorname{lnt}-1.106$ & 0.9645 \\
\hline
\end{tabular}

DS, decursin species; RT, root; FL, flower; LDH, layered double hydroxide; PBS, Phosphate buffered saline; HBSS, Hanks' balanced salt solution.

the molecular arrangement of the AGN extract was re-organized by host LDH nanostructures. Through FT-IR spectra, organic moieties such as carbohydrate and DS in both extract preserved their intact structure after hybridization. The hydrodynamic radii (Supplementary Figures $\mathrm{S} 1 \mathrm{~B}, \mathrm{C}$ ) and primary particle size (Figures 4c,d) of both hybrids showed that several LDH particles might be assembled without serious aggregation. A sand-rose morphology consisting of randomly stacked layers of LDH was also found in a previous study when organic moieties of a natural extract were incorporated into LDH nanostructures (Kim et al., 2016). Random stacking of thin LDH layers $(\sim 19.4 \mathrm{~nm}$ thickness from Figure 4) could form pores able to accommodate the extract. Zeta potential results also supported the conclusion that AGN extract was trapped inside the nanostructures. Zeta potential values of both RT-LDH and FL-LDH were close to those of pristine $\mathrm{LDH}$, showing positive values (Figure 5). If the AGN extract covered only the surfaces of LDH nanostructures, the surface charge of the hybrids would be closer to that of AGN extract. Thus, we suggest that LDHs accommodated organic moieties inside the inter-particle pores rather than at its surface. Although LDH can accommodate negatively charged molecules in its interlayer space, we excluded this possibility because the XRD pattern of the hybrid (Figure 2) did not show significant interlayer expansion [(003) peak position].

The contents of AGN extract in the hybrid were 18.4 and 16.1 wt/wt $\%$ for RT-LDH and FL-LDH, respectively. These contents were larger than those reported in our previous study, where we incorporated AGN root extract into $\mathrm{LDH}$ utilizing methanol solvent (Kim et al., 2016). We are fairly sure that the DMSO solvent played an important role in AGN incorporation; it did not only solubilize as much AGN as possible but also guided them to the LDH nanostructure resulting in efficient hybridization. It was worthy to note that the DS content of extract in hybrids was dramatically enhanced compared with the DS content in the extract itself, showing 62.8 and $13.8 \mathrm{wt} / \mathrm{wt} \%$ in RT-LDH
TABLE 4 | Parameters obtained from kinetic fitting results of the Elovich equation for the release of DS from AGN-LDH hybrids in $\mathrm{pH}$ 7.4 PBS or pH 4.5 HBSS.

\begin{tabular}{lcl}
\hline & $\boldsymbol{a}$ & \multicolumn{1}{c}{$\boldsymbol{b}$} \\
\hline RT-LDH in pH 7.4 PBS & -0.0539 & 0.738 \\
RT-LDH in pH 4.5 HBSS & 6.21 & 3.26 \\
FL-LDH in pH 7.4 PBS & -0.142 & 0.00784 \\
FL-LDH in pH 4.5 HBSS & -0.407 & 0.509 \\
\hline
\end{tabular}

DS, decursin species; AGN, Angelica gigas Nakai; $L D H$, layered double hydroxide; PBS, Phosphate buffered saline; HBSS, Hanks' balanced salt solution; RT, root; $F L$, flower.

and FL-LDH hybrids, respectively. This result indicates that $62.8 \%$ of total organic moiety in the RT-LDH hybrid $(13.8 \%$ for FL-LDH) was DS, which has anticancer activity. Therefore, we expect that both hybrids would exhibit high anticancer efficacy as nanomedicines.

Both hybrids showed DS release patterns fitted to the Elovich model, which explained adsorption or desorption of adsorbate mediated by bulk surface diffusion. According to the literature, organic molecules encapsulated in LDHs followed the power function model, which hypothesized release mediated by flat surface diffusion or ion exchange (Kang et al., 2015). The Elovich model in this study suggested that the DS moiety was located in the 3-dimensional inter-particle space rather than being arranged in the flat interlayer space. This point is important for anticancer drug release from nanoparticles as the arrangement affects drug release kinetics. When drug molecules are located as a quasi-bulk state in large inter-particle pores as in current sample, a large amount of drug moiety can be easily released to act as anticancer agents. Thus, incorporation of DS moieties in the inter-particle space in the present hybrids and Elovich release could be advantageous to anticancer nanomedicine. It was also noteworthy that the both RT-LDH and FL-LDH hybrids showed enhanced drug release in $\mathrm{pH}$ 4.5 HBSS solution, which simulates 

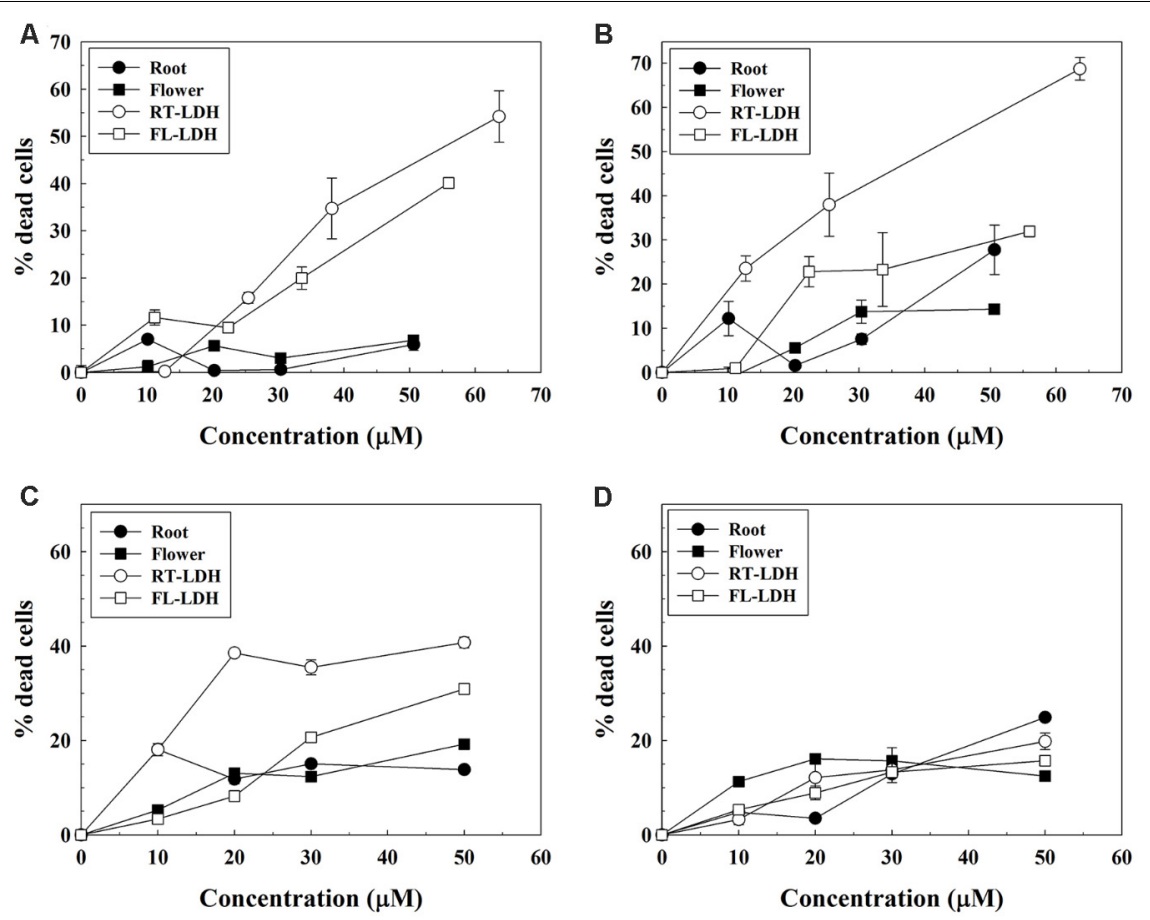

FIGURE 7 | Trypan blue assay of AGN extracts and AGN-LDH hybrids in (A) A549, (B) HeLa, (C) HEK293T, and (D) C2C12 cells. X-axis means DS concentration. AGN, Angelica gigas Nakai; LDH, layered double hydroxide.

the lysosomal condition in cancer cells. In this condition, $\mathrm{LDH}$ can be partially dissolved into simple ions, thus release of loaded drug is accelerated. As it is known that drug-LDH hybrids are internalized into cells via endocytosis (Oh et al., 2006a), lysosomal escape of DS moiety from both RT-LDH and FL$\mathrm{LDH}$ hybrids is expected with increased DS amount compared with simulated physiological condition (PBS). FL-LDH show suppressed DS release amount in both $\mathrm{pH} 7.4 \mathrm{PBS}$ and $\mathrm{pH}$ 4.5 HBSS compared with RT-LDH, possibly due to the higher organic moieties content in FL-LDH than in RT-LDH (13.8\% DS and $86.2 \%$ of other organic moieties, respectively, in FL-LDH compared with $62.8 \%$ DS and $37.2 \%$ in RT-LDH). Major organic components such as lipid, carbohydrate, etc. are thought to trap DS moiety and retard its release. Nevertheless, we conducted anticancer assays for both hybrids, expecting enhanced DS release from the both hybrid in lysosomal condition.

From the literature, DS is known to inhibit cell cycle progression and induce apoptosis in human prostate and murine colon cancer cells (Yim et al., 2005; Son et al., 2011). In addition, decursin and decursinol angelate have been reported to have anti-cancer effects against human cervical cancer cells but not lung cancer cells (Yim et al., 2011). We used human cervical (HeLa), lung (A549), and embryonic kidney cancer cells (HEK293T) for this assay in order to examine the anti-cancer activity of decursin-containing AGN$\mathrm{LDH}$ hybrids on various cell types. Anticancer efficacy test results (Figure 7) showing higher percentages of dead cells for treatment with hybrids than for extract only, could be easily explained by a more efficient cellular uptake mechanism and facilitated release of DS in the lysosomal condition. Antiproliferating activity of AGN-LDH was also examined in $\mathrm{C} 2 \mathrm{C} 12$ myoblast cells (Figure 7D). It was known that non-transformed "normal" cells were less sensitive to DS. This low cytotoxicity of AGN-LDH in $\mathrm{C} 2 \mathrm{C} 12$ cells displays its safety to normal cells. Extracts showed relatively high anti-cancer activity in HeLa cells compared with A549 cells, which is consistent with the results of a previous report (Yim et al., 2011). However, interestingly, LDH hybrids showed significant anticancer activity in all cell lines, depending on the concentration used. Decursin species in extract (closed circles in Figure 7) are thought to penetrate the cell membrane by passive diffusion or facilitated transport. However, DS can massively enter cancer cells via LDHassisted endocytosis and can be released to the cytosol from LDH in acidic lysosomal conditions. In addition, A549 cells result suggests that cytosolic delivery of DS by encapsulation into LDH nanocarriers also may avoid cellular efflux system, contrary to DS only. It should be noted that the amounts of DS in both hybrid and extract were set to be almost the same, and that LDH itself is known to be highly compatible with various cancer cell lines (Choi et al., 2007, 2009). In Supplementary Figure S2, minimal cytotoxicity of LDH itself was also confirmed by MTT assay. Thus, the enhanced anticancer efficacy of hybrids was thought to be attributed to the hybridization of DS with LDHs, as reported previously for drug-LDH hybrids (Oh et al., 2006c; Kim et al., 2013, 2014), and this study verified that the drug-LDH strategy as a nanomedicine can be applied to a natural extract. The fate of $\mathrm{LDH}$, during and after delivering therapeutic agents to cells, is not 
fully comprehended; however, we can suggest several processes according to the literatures. In our previous work based on electron microscopy (tracing LDH part) and confocal microscopy (tracing encapsulated drug molecules), drug encapsulated LDH enters cell, releases drugs to the cytosol and is excreted out of the cell after $24 \mathrm{~h}$ (Oh et al., 2011). The other literature also reported that LDHs could be taken up by cells through endocytosis and finally exocytosed depending on particle size (Chung et al., 2012). The report also claimed that LDH particles could be degraded in lysosome which fused with LDH-containing endosome. To summarize, once entered $\mathrm{LDH}$ undergoes partial degradation and exocytosis. Between the two hybrids investigated, RT-LDH showed higher cancer cell suppression effects than did FL-LDH, even though the same quantities of DS were present in both hybrids. This result can be explained by the release behaviors; RT-LDH exhibited $\sim 35 \%$ release within 120 min, while FL-LDH released $\sim 18 \%$ in this same amount of time. Thus, facilitated DS release from RT-LDH in the lysosomal condition is thought to induce effective anticancer activity.

\section{CONCLUSION}

We prepared hybrids between $\mathrm{LDH}$ and AGN root or flower extract containing DS, anticancer phytochemicals, via the reconstruction route. In order to effectively solubilize DS and to facilitate hybridization with $\mathrm{LDH}$, polar aprotic DMSO solvent was used. According to the XRD patterns, FT-IR spectra, SEM images, and zeta potential values, the AGN-LDH hybrid possessed AGN extract inside the nanostructured pores developed by random stacking of $\mathrm{LDH}$ nanoparticles. Quantitative analyses revealed that 15-20 wt\% of extract was incorporated into AGN-LDH hybrids, and DS in AGN-LDH hybrid was twice as concentrated as that in AGN extract itself. The release profiles of both AGN-LDH in $\mathrm{pH} 7.4$ PBS and $\mathrm{pH}$ 4.5 HBSS fitted well with the Elovich equation, indicating bulk surface release, suggesting that the DS located in the inter-particle pore of $\mathrm{LDH}$ was released in a bulk surface manner. Cell viability tests by trypan blue assay in cancer cells

\section{REFERENCES}

Baumann, L. (2014). Cosmeceuticals and Cosmetic Ingredients. New York, NY: McGraw-Hill Education.

Berber, M. R., Minagawa, K., Katoh, M., Mori, T., and Tanaka, M. (2008). Nanocomposites of 2-arylpropionic acid drugs based on $\mathrm{Mg}-\mathrm{Al}$ layered double hydroxide for dissolution enhancement. Eur. J. Pharm. Sci. 35, 354-360. doi: 10.1016/j.ejps.2008.08.006

Cavani, F., Trifirò, F., and Vaccari, A. (1991). Hydrotalcite-type anionic clays: preparation, properties and applications. Catal. Today 11, 173-301. doi: 10.1016/0920-5861(91)80068-K

Choi, S.-J., Oh, J.-M., and Choy, J.-H. (2009). Toxicological effects of inorganic nanoparticles on human lung cancer A549 cells. J. Inorg. Biochem. 103, 463-471. doi: 10.1016/j.jinorgbio.2008.12.017

Choi, S.-J., Oh, J.-M., Park, T., and Choy, J.-H. (2007). Cellular toxicity of inorganic hydroxide nanoparticles. J. Nanosci. Nanotechnol. 7, 4017-4020. doi: 10.1166/ jnn.2007.085

Chung, H.-E., Park, D.-H., Choy, J.-H., and Choi, S.-J. (2012). Intracellular tracfficking pathway of layered double hydroxide nanoparticles in human cells: demonstrated the dramatically enhanced anticancer activity of the AGN-LDH hybrid compared with AGN itself. Between the two hybrids examined, RT-LDH was determined to have higher anticancer activity than FL-LDH, and this difference was attributed to the facilitated DS release from RT-LDH in the lysosomal condition.

\section{AUTHOR CONTRIBUTIONS}

$\mathrm{H}-\mathrm{JK}$ carried out the preparation, characterization, and release study on AGN-LDH hybrids. GJL performed the anticancer activity tests and interpreted data. A-JC prepared AGN extract and evaluated the solubility and bio-active components of AGN extract. T-HK analyzed characterization data of AGN-LDH hybrids. T-iK designed the anticancer activity evaluation and drafted the manuscript. J-MO designed the material preparation and also drafted the manuscript. All authors read and approved the final manuscript.

\section{FUNDING}

This work was supported by a grant from the Postharvest Research Project (PJ010502) of RDA, Republic of Korea, by Basic Science Research Program through the National Research Foundation of Korea (NRF) funded by the Ministry of Science, ICT, and Future Planning (NRF-2017R1A2B4006352 and NRF-2014R1A1A1037692), and by Promising-Pioneering Researcher Program through Seoul National University (SNU) in 2015. This work was also supported (in part) by the Yonsei University Research Fund of 2018.

\section{SUPPLEMENTARY MATERIAL}

The Supplementary Material for this article can be found online at: https://www.frontiersin.org/articles/10.3389/fphar. 2018.00723/full\#supplementary-material

size-dependent celluar delivery. Appl. Clay Sci. 6, 24-30. doi: 10.1016/j.clay. 2012.06.007

Circosta, C., Pasquale, R. D., Palumbo, D. R., Samperi, S., and Occhiuto, F. (2006). Estrogenic activity of standardized extract of Angelica sinensis. Phytother. Res. 20, 665-669. doi: 10.1002/ptr.1928

Guo, J., Jiang, C., Wang, Z., Lee, H. J., Hu, H., Malewicz, H., et al. (2007). A novel class of pyranocoumarin anti-androgen receptor signaling compounds. Mol. Cancer Ther. 6, 907-917. doi: 10.1158/1535-7163.MCT-06-0231

Hibino, T., and Tsunashima, A. (1998). Characterization of repeatedly reconstructed $\mathrm{Mg}-\mathrm{Al}$ hydrotalcite-like compounds: gradual segregation of aluminum from the structure. Chem. Mater. 10, 4055-4061. doi: 10.1021/ cm980478q

Hu, J., Wu, Z., Yan, J., Pang, W., Liang, D., and Xu, X. (2009). A promising approach for understanding the mechanism of Traditional Chinese Medicine by the aggregation morphology. J. Ethnopharmacol. 123, 267-274. doi: 10.1016/ j.jep.2009.03.007

Jalali, M. (2006). Kinetics of non-exchangeable potassium release and availability in some calcareous soils of western Iran. Geoderma 135, 63-71. doi: 10.1016/j. geoderma.2005.11.006 
Jiang, C., Lee, H.-J., Li, G.-X., Guo, J., Malewicz, B., Zhao, Y., et al. (2006). Potent antiandrogen and androgen receptor activities of an Angelica gigas-containing herbal formulation: identification of decursin as a novel and active compound with implications for prevention and treatment of prostate cancer. Cancer Res. 66, 453-463. doi: 10.1158/0008-5472.CAN-05- 1865

Kang, H., Kim, H.-J., Yang, J.-H., Kim, T.-H., Choi, G., Paek, S.-M., et al. (2015). Intracrystalline structure and release pattern of ferulic acid intercalated into layered double hydroxide through various synthesis routes. Appl. Clay Sci. 11, 32-39. doi: 10.1016/j.clay.2015.04.015

Kesarwani, K., and Gupta, R. (2013). Bioavailability enhancers of herbal origin: an overview. Asian Pac. J. Trop. Biomed. 3, 253-266. doi: 10.1016/S2221-1691(13) 60060-X

Kil, J.-S., Kim, M.-G., Choi, H. M., Lim, J. P., Boo, Y., Kim, E. H., et al. (2008). Inhibitory effects of Angelicae Gigantis Radix on osteoclast formation. Phytother. Res. 22, 472-476. doi: 10.1002/ptr.2342

Kim, H.-J., Ryu, K., Kang, J.-H., Choi, A.-J., Kim, T.-I., and Oh, J.-M. (2013). Anticancer activity of ferulic acid-inorganic nanohybrids synthesized via two different hybridization routes, reconstruction and exfoliation-reassembly. Sci. World J. 2013:421967. doi: 10.1155/2013/421967

Kim, J. Y., Choi, S.-J., Oh, J.-M., Park, T., and Choy, J.-H. (2007). Anticancer druginorganic nanohybrid and its cellular interation. J. Nanosci. Nanotechnol. 7, 3700-3705. doi: 10.1166/jnn.2007.061

Kim, K.-M., Park, C.-B., Choi, A.-J., Choy, J.-H., and Oh, J.-M. (2011). Selective DNA adsorption on layered double hydroxide nanoparticles. Bull. Korean Chem. Soc. 32, 2217-2221. doi: 10.5012/bkcs.2011.32.7.2217

Kim, T.-H., Kim, H.-J., Choi, A.-J., Choi, H.-J., Bae, Y.-S., and Oh, J.-M. (2015). Preparation of nanopowder consisting of Angelica gigas nakai flower extract and anionic nanoclay. Food Eng. Prog. 19, 360-368. doi: 10.13050/foodengprog. 2015.19.4.360

Kim, T.-H., Kim, H.-J., Choi, A.-J., Choi, H.-J., and Oh, J.-M. (2016). Hybridization between natural extract of angelica gias nakai and inorganic nanomaterials of layered double hydroxide via reconstruction reaction. J. Nanosci. Nanotechnol. 16, 1138-1145. doi: 10.1166/jnn.2016.10688

Kim, T.-H., Lee, G. J., Kang, J.-H., Kim, H.-J., Kim, T.-I., and Oh, J.-M. (2014). Anticancer drug-incorporated layered double hydroxide nanohybrids and their enhanced anticancer therapeutic efficacy in combination cancer treatment. Biomed Res. Int. 2014:193401. doi: 10.1155/2014/193401

Lee, H. J., Lee, H. J., Lee, E. O., Lee, J. H., Lee, K. S., Kim, K. H., et al. (2009). In vivo anti-cancer activity of Korean Angelica gigas and its major pyranocoumarin decursin. Am. J. Chin. Med. 37, 127-142. doi: 10.1142/S0192415X09006722

Li, J.-R., Sun, S.-Q., Wang, X.-X., Xu, C.-H., Chen, J.-B., Zhou, Q., et al. (2014). Differentiation of five species of Danggui raw materials by FTIR combined with 2D-COS IR. J. Mol. Struct. 1069, 229-235. doi: 10.1016/j.molstruc.2014.03.067

Lim, J., Kim, I.-H., Kim, H. H., Ahn, K.-S., and Han, H. (2001). Enantioselective syntheses of decursinol angelate and decursin. Tetrahedron Lett. 42, 4001-4003. doi: 10.1016/S0040-4039(01)00642-6

Maincent, P., Le Verge, R., Sado, P., Couvreur, P., and Devissaguet, J.-P. (1986). Disposition kinetics and oral bioavailability of vincamine-loaded polyalkyl cyanoacrylate nanoparticles. J. Pharm. Sci. 75, 955-958. doi: 10.1002/jps. 2600751009

Martínez-Ortiz Mde, J., Lima, E., Lara, V., and Vivar, J. M. (2008). Structural and textural evolution during folding of layers of layered double hydroxides. Langmuir 24, 8904-8911. doi: 10.1021/la801442n

Mengel, K. (2000). Potassium release from mineral structures by $\mathrm{H}+$ ion resin. Geoderma. 96, 291-305. doi: 10.1016/S0016-7061(00)00014-8

Mohammed, A. R., Weston, N., Coombes, A. G. A., Fitzgerald, M., and Perrie, Y. (2004). Liposome formulation of poorly water soluble drugs: optimisation of drug loading and ESEM analysis of stability. Int. J. Pharm. 285, 23-34. doi: 10.1016/j.ijpharm.2004.07.010

Nyambo, C., Chen, D., Su, S., and Wilkie, C. A. (2009). Does organic modification of layered double hydroxides improve the fire performance of PMMA? Polym. Degrad. Stabil. 94, 1298-1306. doi: 10.1016/j.polymdegradstab.2009. 03.023
Oh, J.-M., Choi, S.-J., Kim, S.-T., and Choy, J.-H. (2006a). Cellular uptake mechanism of an inorganic nanovehicle and its drug conjugates: enhanced efficacy due to clathrin-mediated endocytosis. Bioconjugate Chem. 17, 1411-1417. doi: 10.1021/bc0601323

Oh, J.-M., Kwak, S.-Y., and Choy, J.-H. (2006b). Intracrystalline structure of DNA molecules stabilized in the layered double hydroxide. J. Phys. Chem. Solids 67, 1028-1031. doi: 10.1016/j.jpcs.2006.01.080

Oh, J.-M., Park, M., Kim, S.-T., Jung, J.-Y., Kang, Y.-G., and Choy, J.-H. (2006c). Efficient delivery of anticancer drug MTX through MTX-LDH nanohybrid system. J. Phys. Chem. Solids 67, 1024-1027. doi: 10.1016/j.jpcs.2006.01.033

Oh, J. M., Choi, S. J., Lee, G. E., Han, S. H., and Choy, J. H. (2009). Inorganic drugdelivery nanovehicle conjugated with cancer-cell-specific ligand. Adv. Funct. Mater. 19, 1617-1624. doi: 10.1002/adfm.200801127

Oh, J.-M., Park, C.-B., and Choy, J.-H. (2011). Intracellular drug delivery of layered double hydroxide nanoparticels. J. Nanosci. Nanotechnol. 11, 1632-1635. doi: 10.1166/jnn2011.3409

Pérez-Ramírez, J., Abelló, S., and van der Pers, N. M. (2007). Memory effect of activated $\mathrm{Mg}-\mathrm{Al}$ hydrotalcite: in situ XRD studies during decomposition and gas-phase reconstruction. Chem. Eur. J. 13, 870-878. doi: 10.1002/chem. 200600767

Rasoanaivo, P., Wright, C. W., Willcox, M. L., and Gilbert, B. (2011). Whole plant extracts versus single compounds for the treatment of malaria: synergy and positive interactions. Malar. J. 10(Suppl. 1):S4. doi: 10.1186/1475-2875-10S1-S4

Rives, V. (2001). Layered Double Hydroxides: Present and Future. New York, NY: Nova Publishers.

Shariatmadari, H., Shirvani, M., and Jafari, A. (2006). Phosphorus release kinetics and availability in calcareous soils of selected arid and semiarid toposequences. Geoderma 132, 261-272. doi: 10.1016/j.geoderma.2005.05.011

Son, S. H., Park, K. K., Park, S. K., Kim, Y. C., Kim, Y. S., Lee, S. K., et al. (2011). Decursin and decursinol from Angelica gigas inhibit the lung metastasis of murine colon carcinoma. Phytother. Res. 25, 959-964. doi: 10.1002/ptr.3372

Song, S.-H., Seo, B.-I., Kim, H.-K., and Park, J.-H. (2004). The effect of angelicae giantis radix, angelicae acutilobae radix and angelicae sinensis radix extract on hydrocorisone acetate-induced model of blood stasis. Kor. J. Herbol. 19, 13-21.

Strober, W. (2001). Trypan blue exclusion test of cell viability. Curr. Protoc. Immunol. 21, A.3B.1-A.3B.2. doi: 10.1002/0471142735.ima03bs2 1

Suzuki, A., Kagawa, D., Fujii, A., Ochiai, R., Tokimitsu, I., and Saito, I. (2002). Short- and long-term effects of ferulic acid on blood pressure in spontaneously hypertensive rats. Am. J. Hypertens. 15, 351-357.

Yim, D., Singh, R. P., Agarwal, C., Lee, S., Chi, H., and Agarwal, R. (2005). A novel anticancer agent, decursin, induces G1 arrest and apoptosis in human prostate carcinoma cells. Cancer Res. 65, 1035-1044.

Yim, N.-H., Lee, J. H., Cho, W.-K., Yang, M. C., Kwak, D. H., and Ma, J. Y. (2011). Decursin and decursinol angelate from Angelica gigas Nakai induce apoptosis via induction of TRAIL expression on cervical cancer cells. Eur. J. Integr. Med. 3, e299-e307. doi: 10.1016/j.eujim.2011.09.007

Zhang, J., Li, L., Jiang, C., Xing, C., Kim, S. H., and Lü, J. (2012). Anti-cancer and other bioactivities of Korean Angelica gigas Nakai (AGN) and its major pyranocoumarin compounds. Anticancer Agents Med. Chem. 12, 1239-1254. doi: $10.2174 / 187152012803833071$

Conflict of Interest Statement: The authors declare that the research was conducted in the absence of any commercial or financial relationships that could be construed as a potential conflict of interest.

Copyright (c) $2018 \mathrm{Kim}$, Lee, Choi, Kim, Kim and Oh. This is an open-access article distributed under the terms of the Creative Commons Attribution License (CC BY). The use, distribution or reproduction in other forums is permitted, provided the original author(s) and the copyright owner(s) are credited and that the original publication in this journal is cited, in accordance with accepted academic practice. No use, distribution or reproduction is permitted which does not comply with these terms. 